Research article

\title{
Microwave dielectric spectroscopy of pure and mixed aromatic ester solvents
}

\author{
Santiago Aparicio* and Rafael Alcalde
}

\author{
Address: Department of Chemistry, University of Burgos, 09001 Burgos, Spain \\ Email: Santiago Aparicio* - sapar@ubu.es; Rafael Alcalde - ralcalde@ubu.es \\ ${ }^{*}$ Corresponding author
}

Published: 12 February 2008

PMC Physics B 2008, I:4 doi:10.1 186/1754-0429-1-4

This article is available from: http://www.physmathcentral.com//754-0429///4

(C) 2008 Aparicio;

This is an Open Access article distributed under the terms of the Creative Commons Attribution License (http://creativecommons.org/ licenses/by/2.0), which permits unrestricted use, distribution, and reproduction in any medium, provided the original work is properly cited.
Received: 13 November 2007

Accepted: 12 February 2008

\begin{abstract}
The structure and dynamics of pure and mixed liquid aromatic esters is studied through microwave dielectric relaxation spectroscopy in the $200 \mathrm{MHz}-20 \mathrm{GHz}$ frequency range. Methyl, ethyl, propyl and butyl benzoate were studied at $298.15 \mathrm{~K}$ to infer the alkylic chain length effect on solvents properties showing that the relaxational behaviour for the four esters is properly described with a single Debye type function for the considered range. Methylbenzoate behaviour was also analyzed in the $278.15-358.15 \mathrm{~K}$ temperature range to study the temperature effect on their properties. Experiments on phenyl acetate were carried out to analyze the effect of aromatic ring position on fluid structure. We have also considered the properties at $298.15 \mathrm{~K}$ of different methylbenzoate containing mixed fluids with n-hexane, cyclohexane, I-chlorohexane, I-hexanol, hexanoic acid and diisopropyl ether to study the effect of different functional groups on fluid structure upon mixing. All the binary mixtures studied are properly described using a bimodal Debye process in the whole composition range. Data are analyzed in terms of the proposed different species present in the fluids and their evolution with composition for mixtures, and with temperature and chain length for pure esters.
\end{abstract}

PAC CODES: $77.22 . G m, 61.25 . E m, 34.20 . G j$

\section{Background}

Dielectric relaxation spectroscopy, DRS, is a powerful tool to elucidate the structure and dynamics of complex fluids because in many cases offers unique information on these systems [1]. Depending on the nature and characteristics of the fluid under study, DRS may provide relevant data about the thermodynamics, kinetic and structural features of solutions. The strong sensitivity of DRS to intermolecular interactions makes this method a valuable tool to get a deeper insight into the liquid state which properties are mainly governed by these forces. Although DRS is commonly applied in the literature for systems in which hydrogen bonds play a central role, such as water or alcohol containing mixtures [2], it can also be useful for the study of molecular 
dynamics in non-hydrogen bonded fluids [3]. Relaxational behaviour of fluids depends not only upon intra- and intermolecular forces but also on other subtle features such as molecular size and shape, these geometric factors are very important to describe the behaviour of liquid mixtures in which weaker intermolecular forces, mainly of dipolar nature, are present.

Aromatic ester solvents are a remarkable family of fluids with technological applications in very different fields [4]. The detailed knowledge of the molecular level structure of these fluids is required not only to improve the processes in which they are involved but also because of the theoretical importance of these compounds. This group of molecules is characterized by the presence of a polarizable aromatic ring in the vicinity of the dipolar ester group, the combination of both properties, together with their aprotic and hydrophobic character, confer them with a highly selective solvent ability. Previous experimental and theoretical results have showed the complexity of aromatic esters fluids structure, in pure or mixed states, which is governed by several factors: $i$ ) their almost planar shape, although decreasing with increasing alkylic chain length, $i$ ) their dipolar character and iii) their ability to act as hydrogen bonding acceptors with several compounds through the carbonyl oxygen of the molecules [5-11]. Results have showed that a very efficient packing is produced in these pure esters because of their shape, which is showed by their high density [5-11], thus giving rise to a remarkable dipolar ordering because of the favourable interaction among the permanent dipolar moments of the ester molecules in these close packings. Aromatic esters packings are disrupted upon mixing although very different effects arise depending on the nature of the second compounds of the mixture [6]. In order to get a deeper insight into the liquid structure of this group of fluids, we report here a DRS study in the microwave frequency range; according to these experimental results, a better knowledge on their structure and dynamics should be gained, from which a complete picture of the facts that govern their behaviour may be obtained, thus completing our previous studies. This is the first DRS study on aromatic solvents to the best of our knowledge.

The study was organized in a systematic way with several objectives in mind: $i$ ) to analyze the alkylic chain length on the structure and dynamics of pure aromatic esters, thus DRS experiments were carried out for methylbenzoate $(\mathrm{MB})$, ethylbenzoate (EB), propylbenzoate (PB) and butylbenzoate (BB) at $298.15 \mathrm{~K} ; \mathrm{ii}$ ) to study the effect of aromatic ring position related to the ester group, thus experiments were done for phenylacetate (PA) at 298.15 K to compare with MB; iii) to infer the temperature effect on aromatic esters structure, then we have studied $\mathrm{MB}$ in the 278.15 - $358.15 \mathrm{~K}$ temperature range in $10 \mathrm{~K}$ steps; $i v$ ) to study the aromatic esters behaviour upon mixing, hence, experiments were done on several MB containing binary mixtures at 298.15 $\mathrm{K}$ in which the second compounds of the mixtures were carefully selected in order to enable the analysis of the effect of dissimilar molecular geometries or different functional groups on mixtures structure, thus mixtures of MB with n-hexane (HEX), cyclohexane (cHEX), 1-chlorohexane (clHEX), 1-hexanol (HEXol), hexanoic acid (HEXac) and diisopropylether (ISOP) were studied. 


\section{Methods}

MB (99.9\% GC purity), EB (99.9\%), PB (99.1\%), BB (99.1\%), PA (99.0\%), HEX (99.7\%), cHEX (99.5\%), clHEX (99.5\%), HEXol (99.5\%), HEXac (99.5\%), and ISOP (99.1\%) were purchased from Fluka and Aldrich and used without further purification. The liquids were degassed with ultrasound for several days before use, and kept out of the light over Fluka Union Carbide 0.4 $\mathrm{nm}$ molecular sieves. Mixtures were prepared into suitably stoppered vials by weighing amounts using a Mettler AT261 balance $\left( \pm 1 \cdot 10^{-5} \mathrm{~g}\right)$; hence, mixtures in the whole composition range were prepared to $\pm 1 \cdot 10^{-4}$ in mole fraction.

Microwave dielectric spectroscopy measurements were carried out according to a coaxial reflection technique using a vector network analyzer (Agilent N5230A) in the $200 \mathrm{MHz}-20 \mathrm{GHz}$ frequency range with an Agilent 85070E dielectric probe kit and an Agilent N4691B ECal electronic calibration module. All the measurements were carried out at $298.15 \mathrm{~K}$ with the temperature controlled through an external circulating bath and measured to $\pm 0.01 \mathrm{~K}$ with a platinum resistance thermometer calibrated to well-known traceable procedures. The apparatus was first calibrated using air, a short-circuit and water at $298.15 \mathrm{~K}$; before each measurement, a refreshment of the calibration was done with the ECal module. Hence, the accuracy of dielectric measurement is estimated to be \pm 0.1 .

\section{Results and Discussion}

The frequency, $v$, dependence of the real, $\varepsilon^{\prime}$, and imaginary, $\varepsilon^{\prime \prime}$, parts of the complex dielectric function, $\varepsilon^{*}$, for the different studied pure and mixed fluids are fitted simultaneously to a relaxation time distribution involving $k$ Debye processes, eq 1.

$$
\varepsilon^{*}(v)=\sum_{i=1}^{k} \frac{\Delta \varepsilon_{i}}{1+i 2 \pi \nu \tau_{i}}+\varepsilon_{\infty}
$$

where $i$ is the imaginary unit, $\varepsilon_{\infty}$ is the dielectric constant in the high frequency limit, $\Delta \varepsilon_{i}$ the relaxation amplitude and $\tau_{i}$ the relaxation time. The parameters of eq 1 were obtained by a least squares procedure with the Kramers - Kronig [1] condition being guaranteed during the fitting procedure. The static dielectric constant (value at zero frequency), $\varepsilon_{S^{\prime}}$ may be calculated as

$$
\varepsilon_{S}=\sum_{i=1}^{k} \Delta \varepsilon_{i}+\varepsilon_{\infty}
$$

and the mixing static dielectric constant, $\varepsilon_{S, m i x}$ as

$$
\varepsilon_{S, \text { mix }}=\varepsilon_{S}-\sum_{i=1}^{2} x_{i} \varepsilon_{S, i}
$$


where $\varepsilon_{S}$ stands for the static dielectric constant of the mixture, $\varepsilon_{S, i}$ is that for pure compounds forming the mixture and $x_{i}$ being their mole fraction. Relaxation of pure aromatic esters is properly described with just one Debye process, $k=1$, whereas for mixtures a bi-modal approach is required, $k=2$.

\section{Pure aromatic esters at 298. I5 K. Alkylic chain length and aromatic ring position effects}

The complex dielectric spectra for alkylbenzoates and phenylacetate at $298.15 \mathrm{~K}$ are reported in Fig. 1, the results of experimental data analysis according to a single Debye-type relaxation function are reported in Table 1 and Fig. 2. From Fig. 1 we may conclude that this simple model is able to reproduce accurately the experimental spectra in the studied frequency range.

For alkylbenzoates a single loss peak is observed which intensity decreases with increasing chain length and at the same time it moves to lower frequencies. A stronger change appears on going from $\mathrm{MB}$ to $\mathrm{EB}$ and after that a smoother effect from EB to BB, Fig. 1; this is in agreement with previous results using different experimental and theoretical methods [4]. The static dielectric constant decreases with increasing chain length whereas the relaxation time presents the opposite behaviour. The Kirkwood correlation factor, g, may be calculated from the reported results according to eq 4 [12,13], the values are showed in Fig. 2.

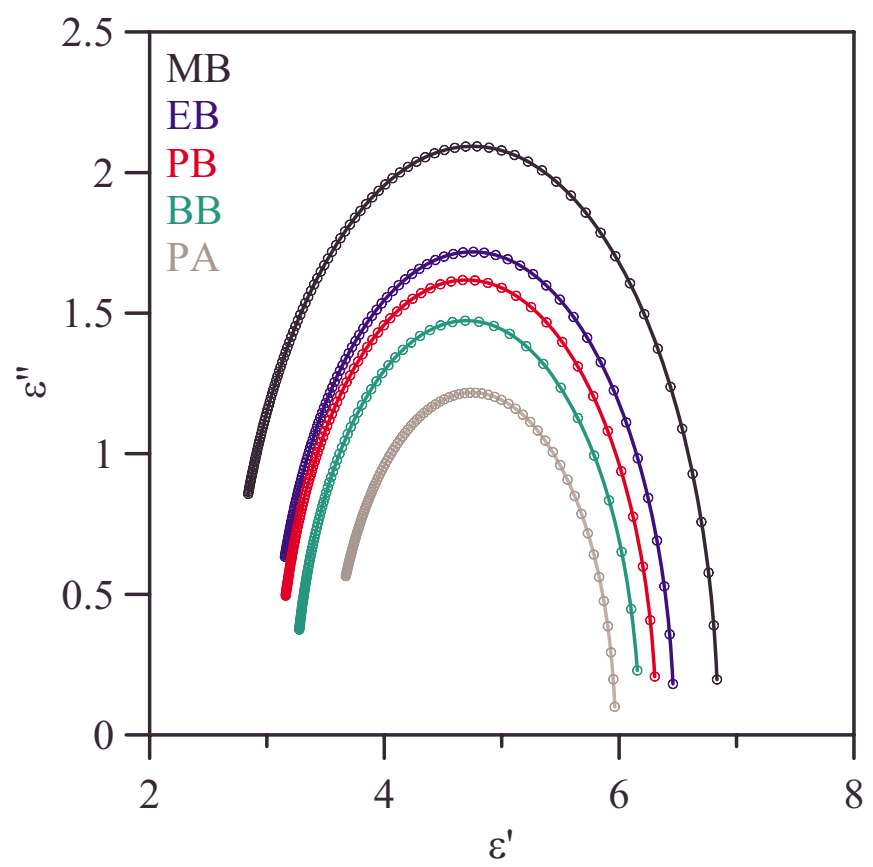

\section{Figure I}

Cole - Cole plots of the complex dielectric function for aromatic esters at $298.15 \mathrm{~K}$. Real, $\varepsilon^{\prime}$, and imaginary, $\varepsilon^{\prime \prime}$, parts. Open circles, experimental points; solid lines, Debye relaxation process with parameters from Table I. 
Table I: Parameterization of experimental dielectric spectra according to a single Debye process for aromatic esters in the $200 \mathrm{MHz}-20 \mathrm{GHz}$ frequency range. Limiting high-frequency permittivity, $\varepsilon_{\infty}$, static dielectric constant, $\varepsilon_{\mathrm{s}}$, and relaxation time, $\tau_{D}$

\begin{tabular}{llllll}
\hline & $T / K$ & $\varepsilon_{\infty}$ & $\varepsilon_{\mathrm{s}}$ & $\tau_{D} / \mathrm{ps}$ & $\chi^{2}$ \\
\hline$M B$ & 278.15 & 2.48 & 7.02 & 53.28 & 0.016 \\
$M B$ & 288.15 & 2.56 & 6.91 & 44.08 & 0.015 \\
$M B$ & 298.15 & 2.66 & 6.84 & 37.49 & 0.015 \\
$M B$ & 308.15 & 2.73 & 6.77 & 32.23 & 0.013 \\
$M B$ & 318.15 & 2.81 & 6.69 & 28.36 & 0.012 \\
$M B$ & 328.15 & 2.89 & 6.61 & 25.45 & 0.010 \\
$M B$ & 338.15 & 2.98 & 6.53 & 23.31 & 0.011 \\
$M B$ & 348.15 & 3.06 & 6.44 & 21.67 & 0.010 \\
$M B$ & 358.15 & 3.16 & 6.35 & 42.03 & 0.008 \\
EB & 298.15 & 3.03 & 6.46 & 51.19 & 0.013 \\
PB & 298.15 & 3.08 & 6.32 & 62.17 & 0.012 \\
PA & 298.15 & 3.23 & 6.17 & 32.65 & 0.012 \\
\hline
\end{tabular}
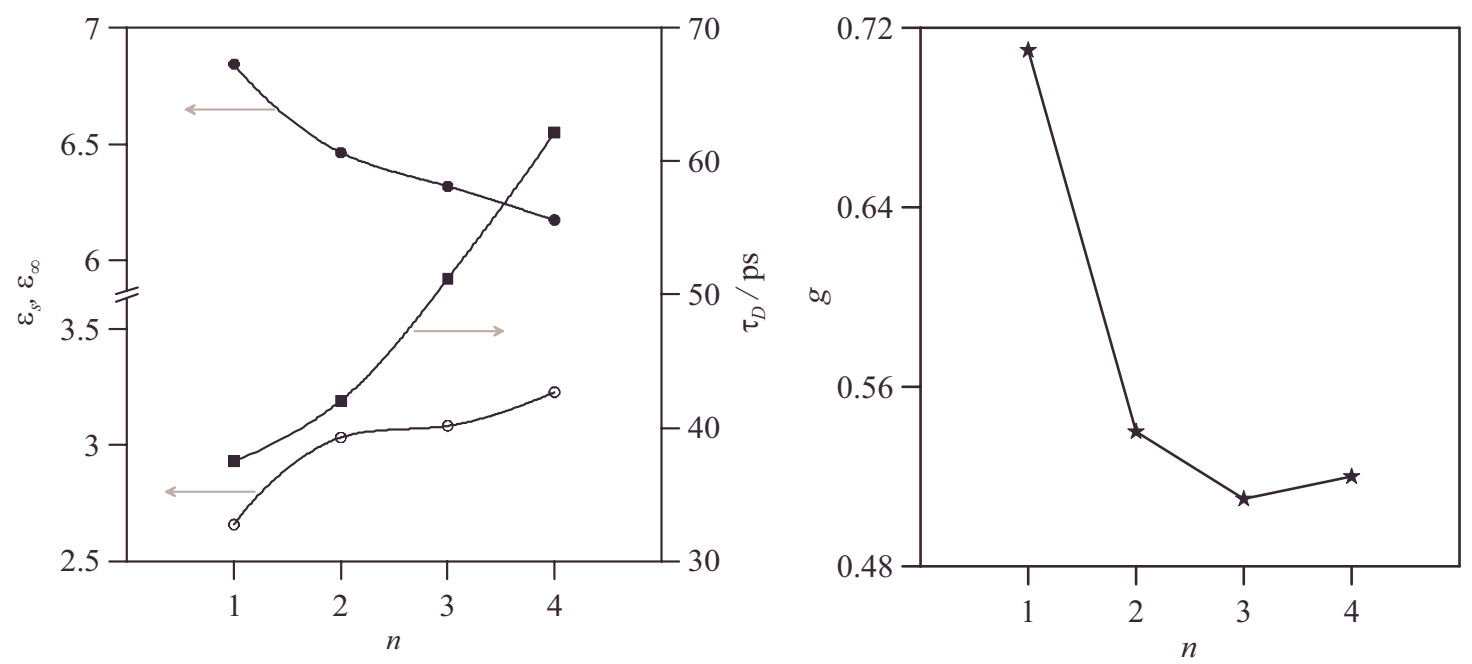

\section{Figure 2}

Limiting high - frequency permittivity, $(\bigcirc) \varepsilon_{\infty}$, static dielectric constant, $(\Theta) \varepsilon_{s}$, relaxation time, $(\square) \tau_{D}$, obtained from the fit of experimental dielectric spectra according to a single Debye process and ( $\star$ ) g, Kirkwood correlation factor for alkylbenzoates at 298.15 K. n, number of carbon atoms in ester alkylic chain. Continuous lines are plotted to show trends. Densities and dipole moments required for $g$ calculation obtained from Refs. [4] and [6]. 


$$
g=\frac{\left(\varepsilon_{S}-\varepsilon_{\infty}\right)\left(2 \varepsilon_{S}+\varepsilon_{\infty}\right)}{\varepsilon_{S}\left(\varepsilon_{\infty}+2\right)^{2}} \frac{9 k_{B} T \varepsilon_{0}}{\mu^{2}} \frac{M}{N_{A} \rho}
$$

where $k_{B}$ and $N_{A}$ are the Boltzmann and Avogadro constants respectively, $T$ is the temperature, $\varepsilon_{0}$ the dielectric constant in vacuum, $\mu$ the dipole moment, $M$ the molar mass and $\rho$ stands for the density. Density and dipole moment required for the calculation of Kirkwood correlation factor are reported in Table 2. The results showed in Fig. 2 show that $g<1$ for all the studied aromatic esters, thus a trend to form associates with anti-parallel dipoles disposition is preferred. In this anti-parallel dipolar arrangement, which is frequently found for almost ellipsoidal molecules such as aromatic esters [14], the molecular dipoles arrange in a parallel way but in opposite directions, thus the dipolar aggregation reduces the dipolar moment giving rise to the so called effective dipole moment, $\mu_{e f f}$, that can be calculated from eq 5 :

$$
g=\left(\frac{\mu_{e f f}}{\mu}\right)^{2}
$$

where $\mu$ is the moment of an isolated molecule (gas phase). In this antiparallel disposition, Fig. 3 , the complex is formed by the interaction among carbonyl and phenyl groups which is very efficient because of the almost planar shape of the molecules. This dipolar arrangement has been previously reported for other families of compounds such as aromatic aldehydes [14]. As the alkylic chain length increases the ellipsoidal character of the molecule also increases which is commonly related with a decrease in $g$ factor, for $\mathrm{MB}$ the dipole moment vector is almost perpendicular to the main molecular axis and then $g$ factor is greater (closer to 1 ) but for longer benzoates the dipole vector moves toward the main molecular axis and then the correlation factor decreases. Anyhow, the correlations factor obtained for the studied alkylbenzoates correspond to fluids with an intermediate degree of self - association. On the other side, we should remark the

Table 2: Experimental density, $\rho$, and calculated (B3LYP/6-3II++g**) gas phase dipole moment, $\mu$, for the pure compounds considered in this work at $298.15 \mathrm{~K}$. These properties are used for the calculation of Kirkwood correlation factor in pure and mixed fluids

\begin{tabular}{lll}
\hline & $\rho / \mathrm{g} \mathrm{cm}^{-3}$ & $\mu / \mathrm{D}$ \\
\hline $\mathrm{MB}$ & $1.08363[10]$ & $1.99[4]$ \\
$\mathrm{EB}$ & $1.04161[10]$ & $2.10[4]$ \\
$\mathrm{PB}$ & $1.01791[10]$ & $2.19[4]$ \\
$\mathrm{BB}$ & $0.99984[10]$ & $2.14[4]$ \\
$\mathrm{PA}$ & $1.07337[10]$ & $1.69[4]$ \\
$\mathrm{HEX}$ & $0.65498[6]$ & 0.00 [this work] \\
CHEX & $0.77354[6]$ & 0.30 [this work] \\
CIHEX & $0.87356[6]$ & 2.57 [this work] \\
HEXol & $0.81520[6]$ & 1.80 [this work] \\
HEXac & $0.92201[6]$ & 1.92 [this work] \\
ISOP & $0.71807[6]$ & 1.33 [this work] \\
\hline
\end{tabular}


very abrupt decrease of $g$ on going from MB to EB (a 23.9\%) whereas from EB to BB $g$ is almost constant $(0.54-0.51)$, this is in agreement with previous results [4] that have showed how the addition of the first methylene group causes the greater effect of the alkylbenzoates structure whereas successive additions produces smoother changes. The decreasing static dielectric constant with increasing chain length, Fig. 2, shows also a remarkable change on going from MB to EB and a smoother one for the remaining benzoates. This decreasing static dielectric constant with chain length points to a weaker interaction among dipoles, although moments for isolated molecules are slightly greater for the longer esters [4], the effective dipolar moment arising from the calculated $g$ factors goes from 1.68 D for MB to 1.54 D for BB. On the other side, apart from the slightly lower effective dipole moments, as the alkylic chain length increases the interaction among these dipoles in the anti-parallel arrangement is also more hindered, as the lower density of longer benzoates show [4], and thus lower dielectric constants may be expected. The relaxation time, Fig. 2, increases with increasing chain length, with a different trend from MB to EB and an almost linear increase from $\mathrm{EB}-\mathrm{BB}$, this is justified because heavier associates are formed which then relax at longer times, this fact is more important than the weaker intermolecular forces as the chain increases which is a fact tending to decrease the relaxation times.

These results show the importance of the interaction among phenyl and carbonyl group in the aromatic esters fluid structure, thus the effect of the aromatic ring position with respect to the ester group in the molecule was inferred from the comparison of MB and PA results. PA has a

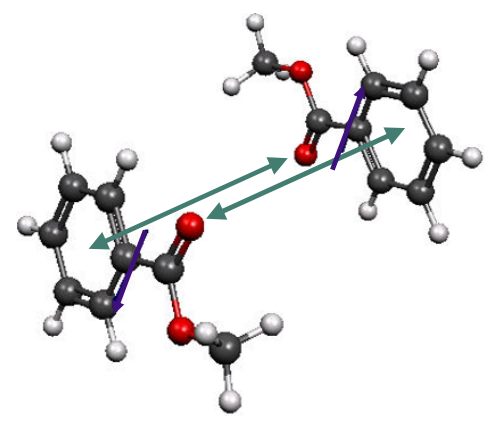

MB

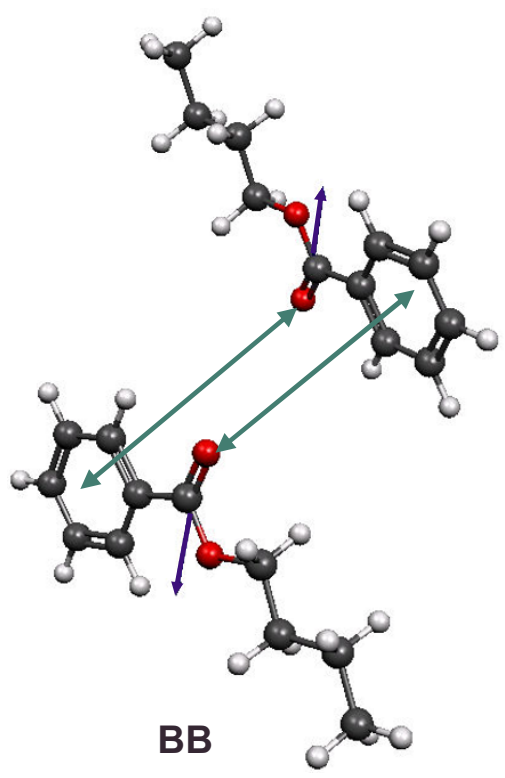

Figure 3

Scheme of the proposed arrangement of alkylbenzoate pairs in anti-parallel disposition. Blue arrows: calculated dipole moments in gas phase [4], green arrows: show the proposed interaction among aromatic ring and carbonyl groups. Atom color code: (red) oxygen, (dark gray) carbon, (soft gray) hydrogen. 
lower value of $g$ factor than $\mathrm{MB}$ ( 0.45 for $\mathrm{PA}, 0.71$ for $\mathrm{MB})$ thus the anti-parallel dipolar disposition is more remarkable. Anyhow, the non-planar shape of PA [4] hinders this arrangement which together with the lower effective dipole moment of PA (1.33) give rise to the lower static dielectric constant for PA. The relaxation time is also lower for PA, Table 1, this fact points to weaker intermolecular dipolar forces in PA. Then, the greater viscosity of PA previously reported [4] is mainly produced by geometric factors arising from its non-planar shape as previously proposed $[4,5]$.

\section{Temperature effect on MB dielectric spectra}

In order to study the temperature effect on aromatic esters structure and dynamics we have carried out DRS measurements in the 278.15 - 358.15 K temperature range, Fig. 4. The obtained spectra are properly described by a single Debye - type function for all the studied temperatures, as we may expect the loss peak moves to higher frequencies, decreasing its intensity, as the temperature rises. The temperature effect on the Kirkwood correlation factor reported in Fig. 5 shows that $g<1$ in the temperature range studied and $(\partial g / \partial T)<0$, thus as the temperature increases the anti-parallel arrangement is reinforced giving rise to lower effective dipolar moments (from 1.73 $\mathrm{D}$ at 278.15 to 1.53 at $358.15 \mathrm{~K}$ ). The packings proposed in Fig. 3 are reinforced with increasing temperature giving rise to more parallel molecular dispositions in the dimmers, decreasing the angle among both molecules involved, in which both dipole moments tend to cancel decreasing the effective dipolar moment. The static dielectric constant reported in Fig. 5 decreases with tem-

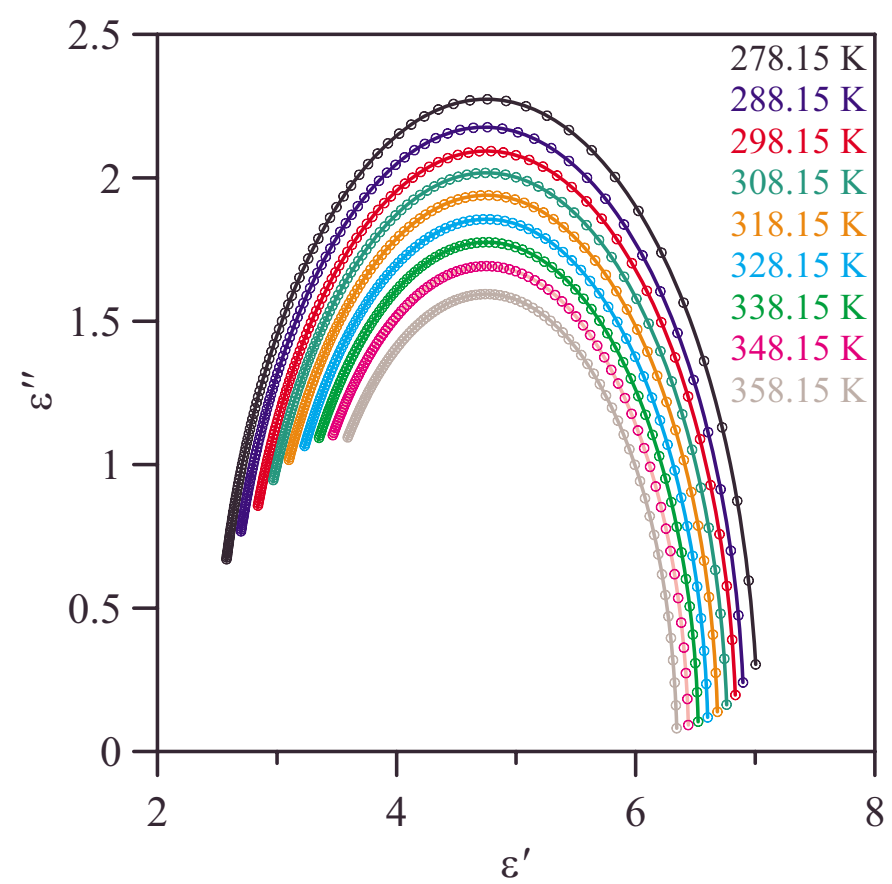

Figure 4

Cole - Cole plots of the complex dielectric function for MB at different temperatures. Real, $\varepsilon^{\prime}$, and imaginary, $\varepsilon^{\prime \prime}$, parts. Open circles, experimental points; solid lines, Debye relaxation process with parameters from Table I. 

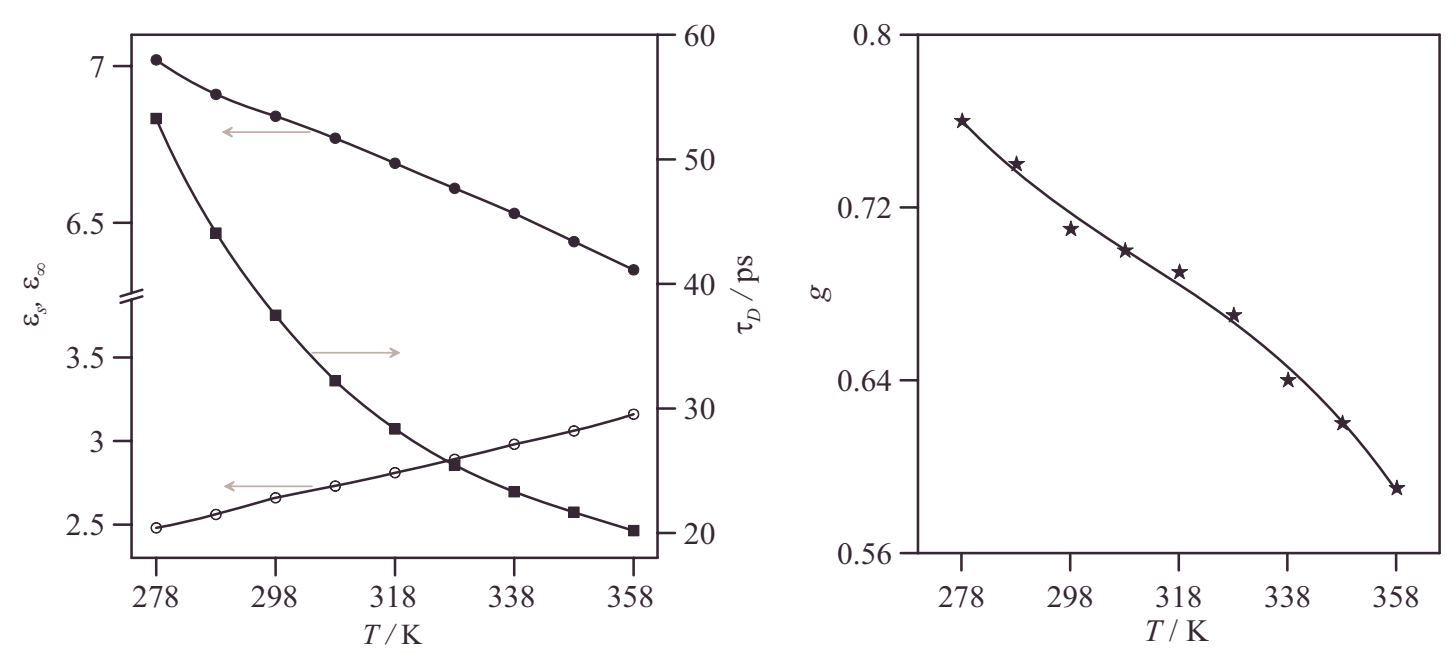

\section{Figure 5}

Limiting high - frequency permittivity, $(\bigcirc) \varepsilon_{\infty}$, static dielectric constant, $(\bullet) \varepsilon_{\mathrm{s}}$, relaxation time, $(\square) \tau_{D}$, obtained from the fit of experimental dielectric spectra according to a single Debye process and $(\star)$ g, Kirkwood correlation factor for $M B$ at different temperatures. Continuous lines are plotted to show trends. For $\tau_{D}$ continuous line shows the Vogel - Fulcher - Tammann fit $\left(\tau_{V F T}^{0}=5.31 \mathrm{ps}, B_{V F T}=249.00 \mathrm{~K}, T_{0}=170.30 \mathrm{~K}\right)$.

perature in an almost linear fashion whereas the dielectric constant in the high frequency limit shows the opposite behaviour which is in agreement with the more efficient anti-parallel arrangements proposed.

The relaxation time reported in Fig. 5 decreases with temperature, the results were tried to be described according to an Eyring approach but great deviations were obtained, thus the temperature dependence of $\mathrm{MB}$ relaxation time was described properly according to the empirical Vogel - Fulcher - Tammann (VFT) approach, eq 6 [15-18]:

$$
\tau=\tau_{V F T}^{0} \exp \left(-B_{V F T} /\left(T-T_{0}\right)\right)
$$

where $T_{0}$ is the Vogel temperature, that use to be $10-30 \mathrm{~K}$ lower than the glass transition temperature. The fitting parameters obtained by a least squares procedure are reported in Fig. 5 . The so - called fragility parameter, $D$, is defined according to eq 7 :

$$
D=\frac{B_{V F T}}{T_{0}}
$$


Thus, the value $D=1.47$ is obtained for $\mathrm{MB}$, this points to a liquid dominated by dipole dipole forces as we may expect, we also may conclude that $\mathrm{MB}$ is a fragile liquid according to the Angell nomenclature [19].

\section{$M B+n-h e x a n e$ mixtures at 298. $15 \mathrm{~K}$}

The results for the MB + HEX mixture at 298.15 K are reported in Figs. 6 and 7. The real and imaginary components of the dielectric spectra decrease with increasing HEX mole fraction with the loss peaks shifting to higher frequencies and decrease their intensities. The low static dielectric constant of the apolar pure HEX justifies the almost planar response obtained for this fluid. The experimental spectra were analyzed according to a bi-modal Debye type relaxation approach, Table 3. The Kirkwood correlation factor for binary mixtures is calculated according to eq 8 $[20,21]$ :

$$
g=\frac{\left(\varepsilon_{S}-\varepsilon_{\infty}\right)\left(2 \varepsilon_{S}+\varepsilon_{\infty}\right)}{\varepsilon_{S}\left(\varepsilon_{\infty}+2\right)^{2}} \frac{9 k T \varepsilon_{0}}{N_{A}} \sum_{i=1}^{2}\left(\phi_{i} \frac{\mu_{i}^{2} \rho_{i}}{M_{i}}\right)^{-1}
$$

where the symbols have the same meaning that in eq 4 , and $\varphi_{i}$ stands for the volume fraction of the $i$ compound of the mixture. The $g$ values reported in Fig. 7 are lower than one for almost the whole composition range but approaching to unity as the HEX mole fraction increases. Hence,

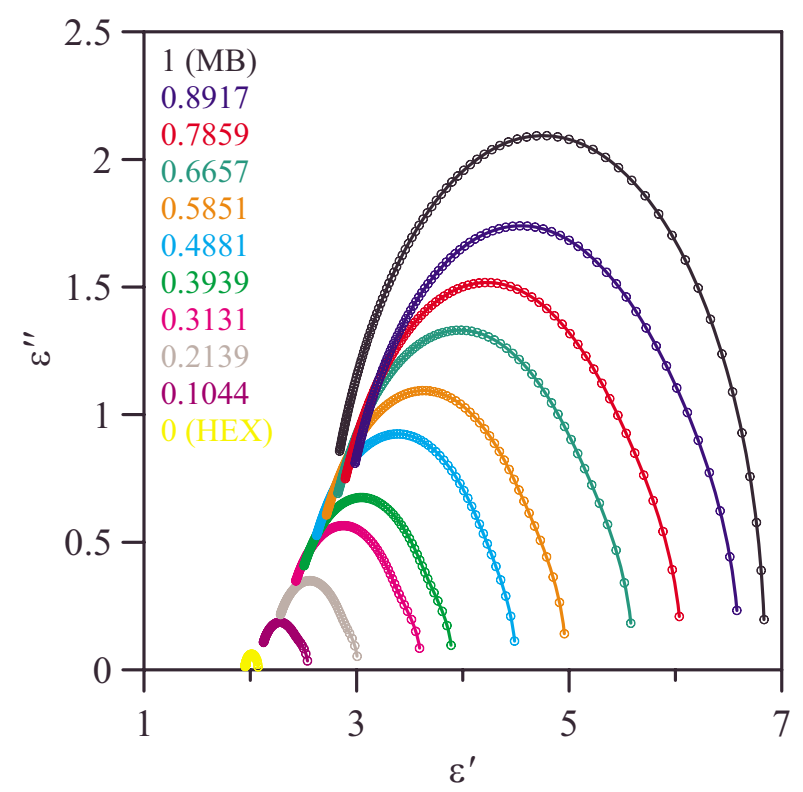

Figure 6

Cole - Cole plots of the complex dielectric function for $x M B+(I-x)$ HEX binary mixtures for different MB mole fractions, $x$, at $298.15 \mathrm{~K}$. Real, $\varepsilon^{\prime}$, and imaginary, $\varepsilon^{\prime \prime}$, parts. Open circles, experimental points; solid lines, Debye relaxation processes (2 Debye processes for mixtures, I Debye process for pure compounds) with parameters from Table 3. 

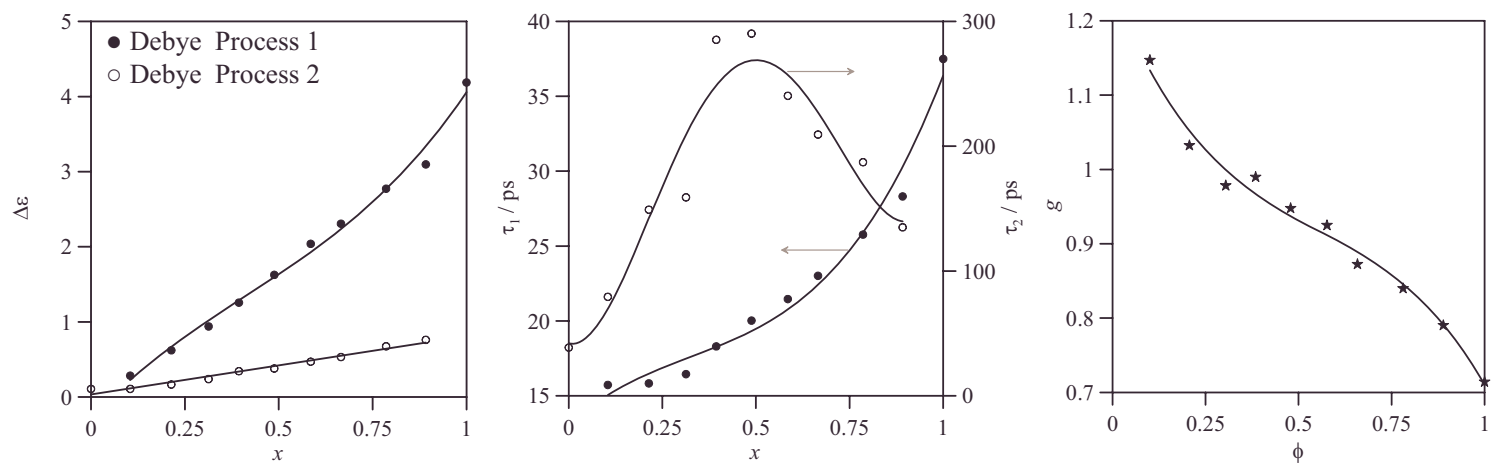

\section{Figure 7}

Dielectric strength, $\Delta \varepsilon$, and relaxation time, $\tau$, obtained from the fit of experimental dielectric spectra according to two Debye processes and Kirkwood correlation factor, g, for $x$ MB + (I - x) HEX binary mixtures at 298.I5 K. Continuous lines are plotted to show trends. Parameters from Table 3. Density data required for $g$ calculation obtained from Ref. 5b. $\varphi=$ MB volume fraction.

the anti-parallel dipolar correlation that prevails in pure $\mathrm{MB}$ is strongly disrupted by the presence of HEX molecules. The obtained correlation factors, very close to unity, discard interaction among HEX and MB molecules. The anti-parallel dipolar disposition may allow the penetration of the almost linear HEX molecules in these structures, thus dipolar interaction among MB dipoles is weakened and dipolar correlation disappears. Hence, as the interaction among HEX and $\mathrm{MB}$ molecules through permanent dipole/induced dipole interactions do not seem to be feasible, geometric effects arising from the permeation of HEX molecules in the MB dipolar arrangement seem to dominate this mixture structure. This is in agreement with our previous thermophysical and theoretical studies [5-11].

The two processes considered for the analysis of MB/HEX dielectric spectra have to be analyzed according to different structural and dynamics facts. The first low frequency process (noted as 1) may be attributed to MB species and it shows the greater amplitude, Fig. 7, whereas the sec-

Table 3: Parameterization of experimental dielectric spectra according to two Debye processes for mixtures and I Debye process for pure fluids for $x \mathrm{MB}+(\mathrm{I}-\mathrm{x}) \mathrm{HEX}$ in the $200 \mathrm{MHz}-20 \mathrm{GHz}$ frequency range at 298. $15 \mathrm{~K}$. Limiting highfrequency permittivity, $\varepsilon_{\infty}$, static dielectric constant, $\varepsilon_{\mathbf{s}}$, Dielectric strength, $\Delta \varepsilon$, and relaxation time, $\tau_{\mathrm{D}}$

\begin{tabular}{lcccccc}
\hline$x$ & $\varepsilon_{\infty}$ & $\Delta \varepsilon_{1}$ & $\Delta \varepsilon_{2}$ & $\tau_{D 1} / \mathrm{ps}$ & $\tau_{\mathrm{D} 2} / \mathrm{ps}$ & $\chi^{2}$ \\
\hline 1 & 2.66 & 4.18 & - & 37.49 & - & 0.015 \\
0.8917 & 2.52 & 3.10 & 0.76 & 28.31 & 134.99 & 0.014 \\
0.7859 & 2.44 & 2.77 & 0.68 & 25.77 & 187.19 & 0.014 \\
0.6657 & 2.33 & 2.30 & 0.53 & 23.01 & 209.33 & 0.014 \\
0.5851 & 2.25 & 2.04 & 0.47 & 21.46 & 240.38 & 0.012 \\
0.4881 & 2.16 & 1.63 & 0.38 & 20.02 & 290.23 & 0.013 \\
0.3939 & 2.09 & 1.26 & 0.34 & 18.30 & 285.28 & 0.011 \\
0.3131 & 2.02 & 0.94 & 0.24 & 16.44 & 159.08 & 0.011 \\
0.2139 & 1.94 & 0.62 & 0.17 & 15.83 & 149.12 & 0.009 \\
0.1044 & 1.84 & 0.28 & 0.11 & 15.72 & 79.30 & 0.006 \\
0 & 1.70 & - & 0.11 & - & 38.65 & 0.004
\end{tabular}


ond higher frequency one (noted as 2) rises from HEX features and it is remarkably smaller than the first one. For pure HEX a very small peak appears, which amplitude is almost null, Table 3, at low frequencies; this peak increases its amplitude with increasing $\mathrm{MB}$ mole fraction with its relaxation time moving remarkably to greater times passing through a maximum. The peak rising from $\mathrm{MB}$ decreases its intensity upon HEX addition with the relaxation time also decreasing, firstly in a steep way and for $x_{\mathrm{MB}}<0.75$ in an almost linear fashion. The mixture behaviour at high $\mathrm{MB}$ mole fractions shows that although HEX molecules disrupts the MB dipolar arrangement, this still prevails for that concentration range, as the positive mixing static dielectric constant reported in Fig. 8 shows; however, the movement of the relaxation time of process 1 to lower frequencies points to a decrease in the size of these aggregates because of the HEX insertion into their dipolar structures. For low MB mole fractions we have the expected behaviour of a diluted polar fluid in an apolar solvent, the amplitude of the peak is almost null and the relaxation time appears at low times pointing to $\mathrm{MB}$ monomers dispersed in the apolar HEX structure, this is confirmed by the negative mixing static dielectric constant for this concentration range, Fig. 8. For HEX molecules, the reported behaviour for process 2 shows the evolution from the almost null relaxation on pure apolar HEX to a process characterized by longer times and more intense amplitudes, thus the permeation of HEX molecules in an increasingly dominated $\mathrm{MB}$ fluid hinders the HEX relaxation probably by geometrical effects rising from its linear shape in comparison with the planar one of MB molecules.

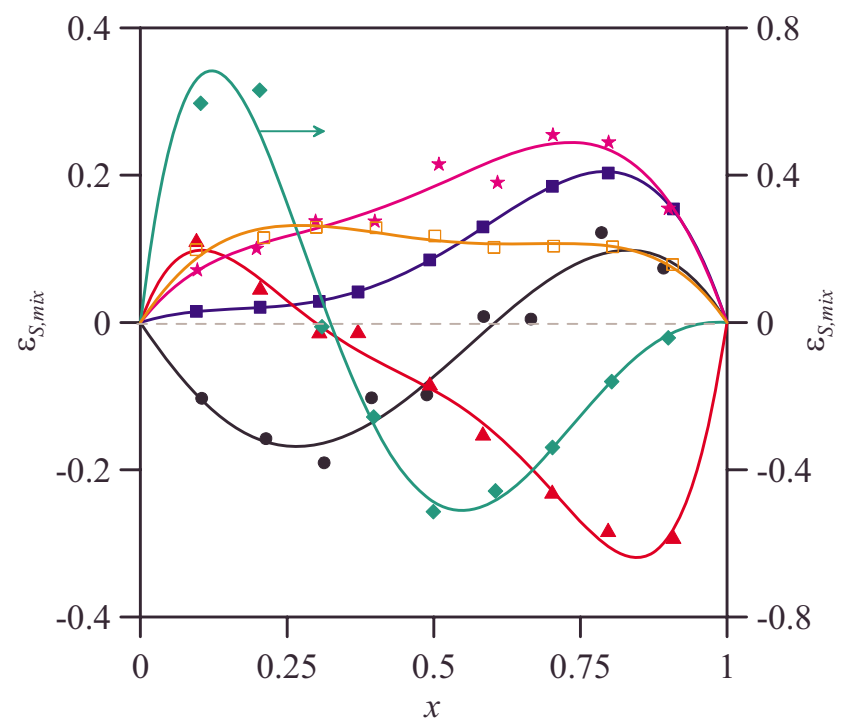

\section{Figure 8}

Mixing static dielectric constant, $\varepsilon_{\mathrm{s}, \text { mix }}$, for $x \mathrm{MB}+(\mathrm{I}-\mathrm{x})$ second compound binary mixtures at $298.15 \mathrm{~K}$. Symbols: experimental points, $(\mathbf{O}) \mathrm{HEX},(\boldsymbol{\square}$, blue) cHEX, $(\boldsymbol{\Lambda}$, red) clHEX, $(\diamond$, green) HEXol, ( $\star$, pink) HEXac, ( $\square$, orange) ISOP; continuous lines: Redlich - Kister fitting. 


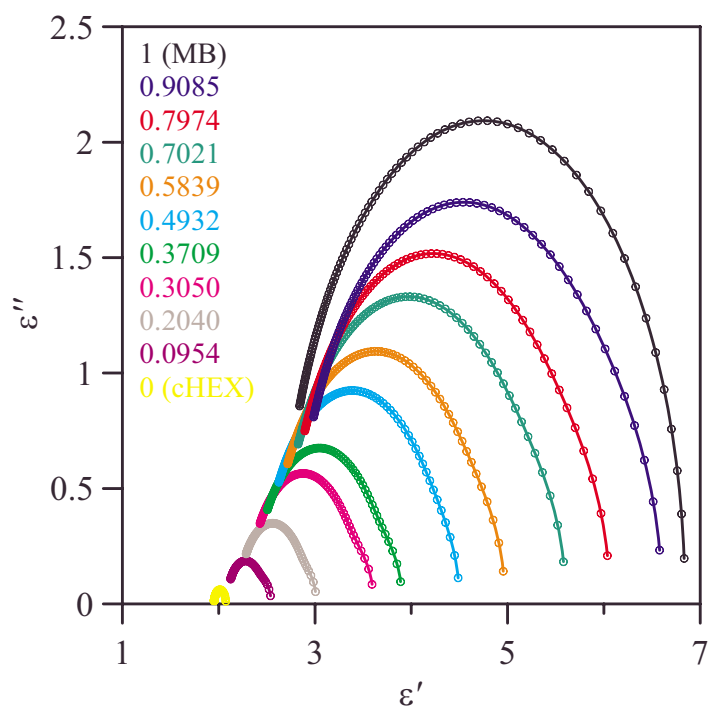

\section{Figure 9}

Cole - Cole plots of the complex dielectric function for $x \mathrm{MB}+(\mathrm{I}-x)$ cHEX binary mixtures for different MB mole fractions, $x$, at $298.15 \mathrm{~K}$. Real, $\varepsilon^{\prime}$, and imaginary, $\varepsilon^{\prime \prime}$, parts. Open circles, experimental points; solid lines, Debye relaxation processes (2 Debye processes for mixtures, I Debye process for pure compounds) with parameters from Table 4.

\section{MB + cyclohexane at 298. I5 K}

The behaviour of these binary mixtures is very similar to that reported in the previous section for HEX containing ones, Fig. 9. The relaxation behaviour of pure cHEX is characteristic of its low dipole moment with a relaxation time greater than the one for pure HEX, because of the cHEX globular shape, and with a static dielectric constant just slightly greater than the one for the linear
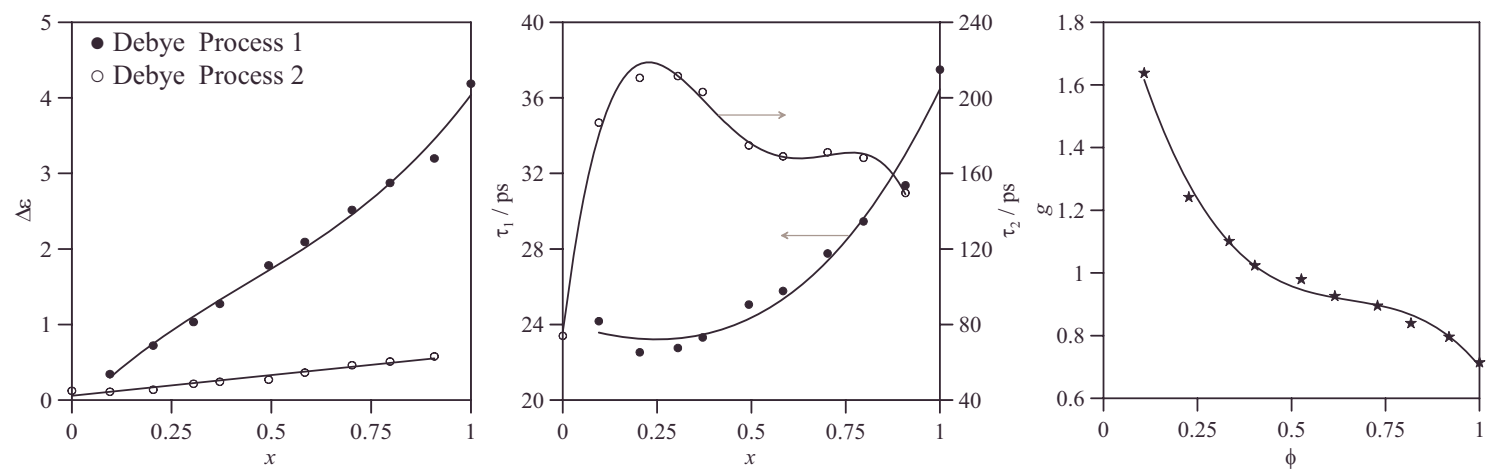

\section{Figure 10}

Dielectric strength, $\Delta \varepsilon$, and relaxation time, $\tau$, obtained from the fit of experimental dielectric spectra according to two Debye processes and Kirkwood correlation factor, g, for $x \mathrm{MB}+(\mathrm{I}-x)$ cHEX binary mixtures at $298.15 \mathrm{~K}$. Continuous lines are plotted to show trends. Parameters from Table 4. Density data required for $g$ calculation obtained from Ref. [6]. $\varphi=$ MB volume fraction. 
alkane. The results of spectra analysis according to the bi-modal Debye relaxation behaviour are reported in Fig. 10. The Kirkwood correlation factor is lower than 1 for MB volume fractions greater than 0.5 , and for this concentration range it increases smoothly in an almost linear fashion whereas for lower $\mathrm{MB}$ concentration it shows a steep increment. The antiparallel dipolar arrangement developed in pure $\mathrm{MB}$ is strongly disrupted by the presence of the almost apolar cHEX molecules $\left(\mu_{298.15 \mathrm{k}}=0.3 \mathrm{D}\right.$, Table 2$)$ [22]; at low MB concentration the $g$ factor is clearly greater than 1, pointing to a parallel dipolar arrangement. The cyclic shape of cHEX molecules allows an effective permeation of the $\mathrm{MB}$ dipolar structures, this permeation has a clearly disruptive effect on the MB dipolar local ordering because of the globular shape of cHEX [23], thus decreasing the stability of antiparallel arrangements (because it hinders the interaction among aromatic rings and ester groups). Hence, as the cHEX concentration increases, $\mathrm{MB}$ molecules are diluted in a cHEX dominated structure and thus dipoles tend to be in a parallel way $(g>1)$ because the antiparallel arrangement is not favoured.

The two relaxation processes (Table 4) can be attributed as in the previous section to $\mathrm{MB}$ and cHEX features (process 1 and 2 respectively). The peak rising from MB decreases its amplitude upon cHEX addition, its relaxation time moving toward lower times shows the disruption of $\mathrm{MB}$ dipolar ordering because of the addition of cycloalkane molecules; thus, and increasing fraction of monomers, relaxing at lower times, justifies the experimental behaviour. The peak attributed to cHEX features increases its intensity in an almost linear way with MB addition, the relaxation time also passes through a maximum pointing to a permeation of cHEX in an MB dominated structure, this permeation seems to hinder the cHEX relaxation. The mixing static dielectric constant reported in Fig. 8 is positive in the whole composition range although for $\mathrm{x}_{\mathrm{MB}}<0.5$ it is almost null, the values for this property at high $\mathrm{MB}$ concentration are slightly greater than the ones for HEX mixtures. This fact shows that in spite of the greater disruption ability of globular cHEX molecules in comparison with the linear HEX ones, the dipolar ordering is slightly greater in cHEX containing mixtures, this is also showed by the results reported in Table 4 that show greater amplitudes and relaxation times for the second process in cHEX mixtures. Thus a certain

Table 4: Parameterization of experimental dielectric spectra according to two Debye processes for mixtures and I Debye process for pure fluids for $x \mathrm{MB}+(I-x) \mathrm{cHEX}$ in the $200 \mathrm{MHz}-20 \mathrm{GHz}$ frequency range at 298. $15 \mathrm{~K}$. Limiting highfrequency permittivity, $\varepsilon_{\infty}$, static dielectric constant, $\varepsilon_{\mathbf{s}}$, Dielectric strength, $\Delta \varepsilon$, and relaxation time, $\tau_{\mathrm{D}}$

\begin{tabular}{lcccccc}
\hline$x$ & $\varepsilon_{\infty}$ & $\Delta \varepsilon_{1}$ & $\Delta \varepsilon_{2}$ & $\tau_{D 1} / \mathrm{ps}$ & $\tau_{\mathrm{D} 2} / \mathrm{ps}$ & $\chi^{2}$ \\
\hline 1 & 2.66 & 4.18 & - & 37.49 & - & 0.015 \\
0.9085 & 2.78 & 3.20 & 0.58 & 31.37 & 149.65 & 0.015 \\
0.7974 & 2.70 & 2.87 & 0.51 & 29.46 & 168.25 & 0.013 \\
0.7021 & 2.63 & 2.52 & 0.46 & 27.76 & 171.19 & 0.014 \\
0.5839 & 2.53 & 2.09 & 0.36 & 25.78 & 169.04 & 0.011 \\
0.4932 & 2.46 & 1.78 & 0.27 & 25.06 & 174.81 & 0.012 \\
0.3709 & 2.37 & 1.27 & 0.24 & 23.32 & 203.11 & 0.010 \\
0.3050 & 2.31 & 1.03 & 0.22 & 22.76 & 211.52 & 0.008 \\
0.2040 & 2.21 & 0.72 & 0.14 & 22.53 & 210.57 & 0.008 \\
0.0954 & 2.09 & 0.34 & 0.11 & 24.18 & 186.87 & 0.006 \\
0 & 1.95 & - & 0.12 & - & 73.98 & 0.005
\end{tabular}


interaction among the very mild dipole of cHEX and the strongest one of $\mathrm{MB}$, or even through permanent - induced dipolar interactions, may be developed that would partially balance the great disruption effect induced by the globular cycloalkane molecule. Anyhow, these interactions among unlike molecules should be present in a low proportion because no peak could be resolved from the spectra and because previous thermophysical results have showed that the disruption effect of cHEX is the prevailing factor.

\section{$M B+$ I-chlorohexane mixtures at 298. I5 K}

Results for this binary system are reported in Figs. 11-12 and in Table 5. Pure clHEX shows a remarkable relaxational behaviour in the studied frequency range with the maximum of the peak at lower times than in pure MB. This peak is in agreement with its dipole moment close to that of pure MB (Table 2) [24], only the linear shape of clHEX gives rise to a faster relaxation compared with MB. The Kirkwood correlation factor of clHEX is remarkably lower than 1 pointing to a well defined anti-parallel dipolar arrangement, the calculated dipole moment for this molecule reported in Fig. 12 is placed almost along the molecular axis and thus this justifies the low $\mathrm{g}$ factor. The anti-parallel arrangement of neighbour clHEX molecules gives rise to an efficient dipolar interaction with the effective dipole moment remarkably lower than the gas phase one. Upon mixing with $\mathrm{MB}$ the two Debye processes obtained from the experimental data show complementary behaviour. The first process assigned to $\mathrm{MB}$ features decreases its intensity with increasing clHEX mole fraction in an almost linear way whereas its relaxation time increases firstly

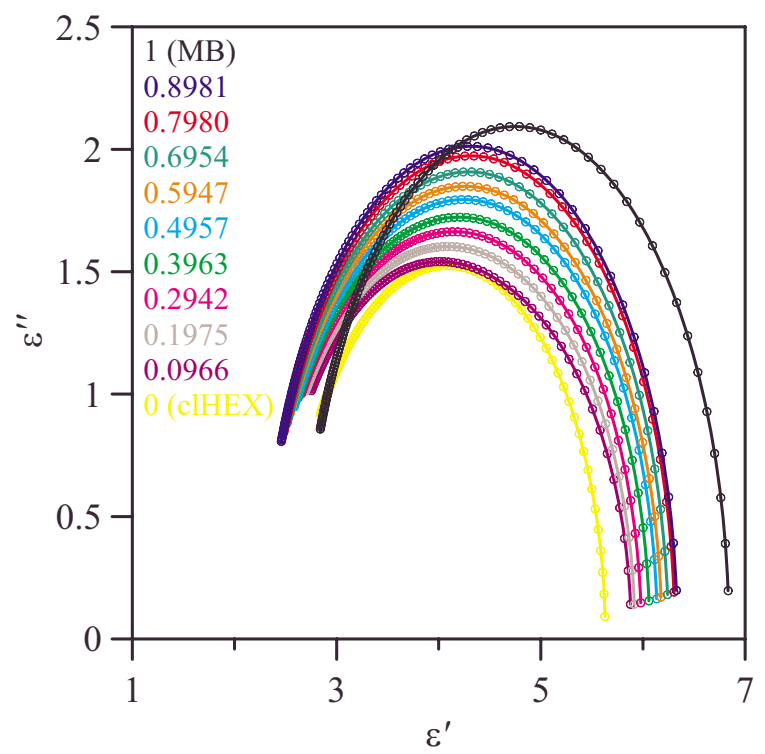

\section{Figure I I}

Cole - Cole plots of the complex dielectric function for $x \mathrm{MB}+(\mathrm{I}-x)$ clHEX binary mixtures for different $\mathrm{MB}$ mole fractions, $x$, at 298. $15 \mathrm{~K}$. Real, $\varepsilon^{\prime}$, and imaginary, $\varepsilon^{\prime \prime}$, parts. Open circles, experimental points; solid lines, Debye relaxation processes (2 Debye processes for mixtures, I Debye process for pure compounds) with parameters from Table 5 . 

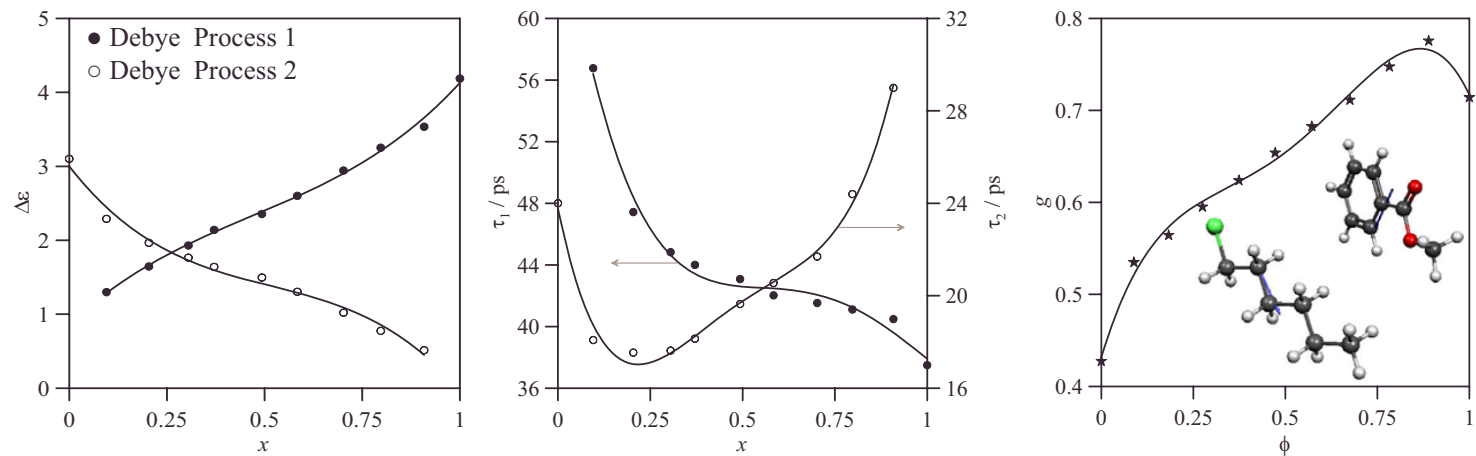

Figure 12

Dielectric strength, $\Delta \varepsilon$, and relaxation time, $\tau$, obtained from the fit of experimental dielectric spectra according to two Debye processes and Kirkwood correlation factor, g, for $x$ MB + (I - x) clHEX binary mixtures at 298. I5 K. Continuous lines are plotted to show trends. Parameters from Table 5. Density data required for $g$ calculation obtained from Ref. [6]. $\varphi=$ MB volume fraction. Molecules inside $g$ factor panel show the gas phase calculated structures of cIHEX and MB (B3LYP/6-3II ++g** theoretical level) for the anti-parallel arrangement of both dipoles. Atom color code as in Fig. 3 with (green) chlorine. Blue arrows, calculated molecular dipole moments.

according to a smooth trend and at low MB concentration in a steep way. As the $\mathrm{g}$ factors reported for these mixtures show, the anti-parallel dipolar arrangement is reinforced upon clHEX addition to $\mathrm{MB}$, hence clHEX disrupt the $\mathrm{MB}$ dipolar structure but its shape allow and efficient packing, as the negative excess molar volume show [6]; thus, although this fact weakens the MB/MB dipolar interaction, as the decreasing amplitude of the first Debye process shows, the new MB/clHEX unlike dipolar interaction in an anti-parallel arrangement, Fig. 12, balance it. The same effect may be inferred form the analysis of the second Debye process assigned to clHEX features, its intensity decreases upon $\mathrm{MB}$ addition almost linearly whereas the relaxation time shows a complex behaviour, firstly decreases in a steep way for very low $\mathrm{MB}$ concentrations and then it increases remarkably. The effect of $\mathrm{MB}$ on clHEX is more remarkable than that of clHEX on MB structure as the results reported in Fig. 12 show. Anyhow, in the mixtures unlike dipolar interac-

Table 5: Parameterization of experimental dielectric spectra according to two Debye processes for mixtures and I Debye process for pure fluids for $x \mathrm{MB}+(\mathrm{I}-\mathrm{x}) \mathrm{clHEX}$ in the $200 \mathrm{MHz}-20 \mathrm{GHz}$ frequency range at 298. I5 K. Limiting highfrequency permittivity, $\varepsilon_{\infty}$, static dielectric constant, $\varepsilon_{\mathbf{s}}$, Dielectric strength, $\Delta \varepsilon$, and relaxation time, $\tau_{\mathrm{D}}$

\begin{tabular}{ccccccc}
\hline$x$ & $\varepsilon_{\infty}$ & $\Delta \varepsilon_{1}$ & $\Delta \varepsilon_{2}$ & $\tau_{D 1} / \mathrm{ps}$ & $\tau_{\mathrm{D} 2} / \mathrm{Ps}$ & $\chi^{2}$ \\
\hline 1 & 2.66 & 4.18 & - & 37.49 & - & 0.015 \\
0.8981 & 2.29 & 3.53 & 0.51 & 40.48 & 28.99 & 0.016 \\
0.7980 & 2.29 & 3.25 & 0.78 & 41.11 & 24.40 & 0.015 \\
0.6954 & 2.29 & 2.94 & 1.02 & 41.53 & 21.69 & 0.014 \\
0.5947 & 2.28 & 2.60 & 1.30 & 42.02 & 20.55 & 0.013 \\
0.4957 & 2.29 & 2.36 & 1.50 & 43.08 & 19.64 & 0.014 \\
0.3963 & 2.29 & 2.14 & 1.64 & 44.00 & 18.14 & 0.012 \\
0.2942 & 2.29 & 1.93 & 1.77 & 44.83 & 17.63 & 0.013 \\
0.1975 & 2.31 & 1.65 & 1.97 & 47.43 & 17.54 & 0.012 \\
0.0966 & 2.36 & 1.30 & 2.29 & 56.77 & 18.08 & 0.012 \\
0 & 2.53 & - & 3.10 & - & 24.00 & 0.011
\end{tabular}


tions are clearly developed, the almost equal magnitude of dipole moments of both molecules favours this fact, upon mixing one type of molecules is changed by the other one, both ones fitting in efficient packings, with their dipoles in anti-parallel arrangements, thus the evolution from like type dipolar arrangements to unlike type ones evolves without losing efficiency in the interactions. Only facts arising from the different shape of both molecules may interfere this process, MB cyclic molecules are poorly fitted in a clHEX dominated structure, as the decrease in the second Debye relaxation process for low $\mathrm{MB}$ concentration shows, whereas upon increasing $\mathrm{MB}$ concentration the fit of both molecules is more efficient and so is the dipolar interaction. The increasing relaxation times for both processes points to a decreasing fraction of monomers in the solution and also to heavier complexes which relax at longer times. The mixing static dielectric constant reported in Fig. 8 is negative in almost the full composition range, only at high clHEX mole fractions it is slightly positive; this is in agreement with the $\mathrm{g}$ values remarkably lower than one arising form the unlike dipolar interaction, this give rise to decreasing values of the effective dipole moment and thus to a lower static dielectric constant upon mixing.

\section{MB + I-hexanol mixtures at 298. I5 K}

Results for this binary system are reported in Figs. 13-14 and in Table 6. Dielectric spectra for pure HEXol is not fully included in the spectral range studied in this work, as we can see in Fig. 13 the maximum of the relaxation peak appears close to the lower frequency limit of our apparatus. Anyhow, the relaxational spectrum of this molecule has been widely studied in the litera-

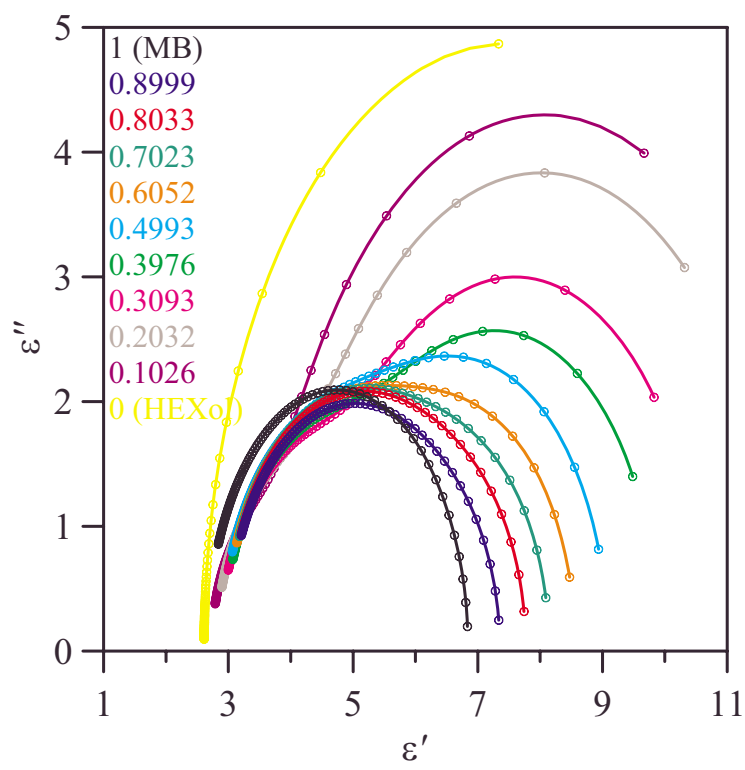

\section{Figure 13}

Cole - Cole plots of the complex dielectric function for $x \mathrm{MB}+(\mathrm{I}-x)$ HEXol binary mixtures for different $M B$ mole fractions, $x$, at $298.15 \mathrm{~K}$. Real, $\varepsilon^{\prime}$, and imaginary, $\varepsilon^{\prime \prime}$, parts. Open circles, experimental points; solid lines, Debye relaxation processes ( 2 Debye processes for mixtures, I Debye process for pure compounds) with parameters from Table 6. 

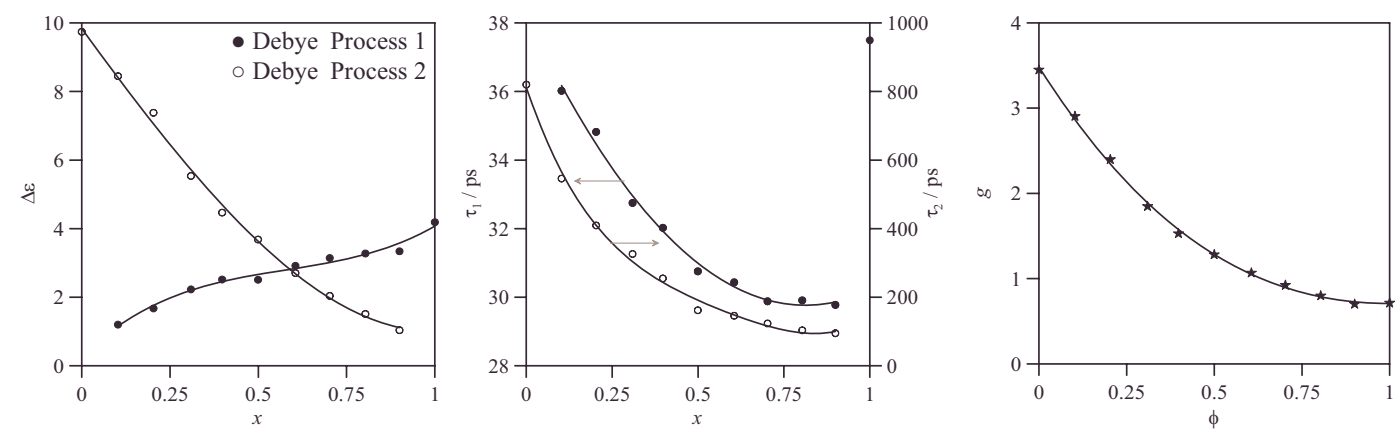

\section{Figure 14}

Dielectric strength, $\Delta \varepsilon$, and relaxation time, $\tau$, obtained from the fit of experimental dielectric spectra according to two Debye processes and Kirkwood correlation factor, $g$, for $x \mathrm{MB}+(I-x)$ HEXol binary mixtures at 298.15 K. Continuous lines are plotted to show trends. Parameters from Table 6. Density data required for $g$ calculation obtained from Ref. [6]. $\varphi=$ MB volume fraction.

ture [25-28] being properly described by a sum of just two Debye processes [28] one with the greater amplitude at around 824 ps (rising from the orientation of hydrogen bonded groups) [28] and other very weak one at around 35 ps (rising from the monomers rotation and from the motions of terminal molecules in hydrogen bonded associates) [29,30]. Considering that the main contribution to the relaxation of pure HEXol arises from the Debye term at around 824 ps we have fitted our data for pure HEXol to a single Debye function, in order to simplify the analysis of mixture behaviour, using the Debye parameters reported by Petong et al [28] for the dominating low frequency process as initial guesses in the fitting procedure, these are the results reported in Table 6. Kirkwood factor for pure HEXol is remarkably larger than 1, thus a parallel arrangement of dipoles in the alcohol liquid structure is clearly preferred which is in agreement with previous results [28]. For HEXol/MB mixtures the relaxation peak moves to lower frequencies and decreases its amplitude remarkably with increasing MB concentration, Fig. 13. The Kirkwood factor moves toward 1 upon $\mathrm{MB}$ addition, thus the preferred parallel dipolar arrangement of HEXol molecules is weakened in mixtures and thus a lower degree of dipolar ordering in mix-

Table 6: Parameterization of experimental dielectric spectra according to two Debye processes for mixtures and I Debye process for pure fluids for $x \mathrm{MB}+(\mathrm{I}-\mathrm{x}) \mathrm{HEXol}$ in the $200 \mathrm{MHz}-20 \mathrm{GHz}$ frequency range at 298. $15 \mathrm{~K}$. Limiting highfrequency permittivity, $\varepsilon_{\infty}$, static dielectric constant, $\varepsilon_{\mathbf{s}}$, Dielectric strength, $\Delta \varepsilon$, and relaxation time, $\tau_{\mathrm{D}}$

\begin{tabular}{ccccccc}
\hline$x$ & $\varepsilon_{\infty}$ & $\Delta \varepsilon_{1}$ & $\Delta \varepsilon_{2}$ & $\tau_{D 1} / \mathrm{ps}$ & $\tau_{\mathrm{D} 2} / \mathrm{ps}$ & $\chi^{2}$ \\
\hline 1 & 2.66 & 4.18 & - & 37.49 & - & 0.015 \\
0.8999 & 2.97 & 3.34 & 1.04 & 29.78 & 95.15 & 0.016 \\
0.8033 & 2.98 & 3.28 & $1.5 \mathrm{I}$ & 29.91 & 103.57 & 0.017 \\
0.7023 & 2.96 & 3.14 & 2.04 & 29.88 & 123.54 & 0.017 \\
0.6052 & 2.94 & 2.92 & 2.71 & 30.43 & 146.01 & 0.016 \\
0.4993 & 2.89 & 2.51 & 3.68 & 30.76 & 161.92 & 0.018 \\
0.3976 & 2.92 & 2.52 & 4.47 & 32.02 & 254.86 & 0.020 \\
0.3093 & 2.87 & 2.23 & 5.54 & 32.75 & 326.15 & 0.022 \\
0.2032 & 2.81 & 1.67 & 7.38 & 34.82 & 409.35 & 0.021 \\
0.1026 & 2.73 & 1.20 & 8.45 & 36.02 & 546.03 & 0.023 \\
0 & 2.61 & - & 9.74 & - & 820.00 & 0.024
\end{tabular}


tures may be inferred. The first Debye process rising from MB features shows a complex behaviour, Fig. 14, its intensity decreases in an almost linear way upon HEXol addition, this may be justified considering that the preferred anti-parallel arrangement of MB dipoles is clearly disrupted by the HEXol molecules for which a parallel situation is more suitable. The relaxation time of the first Debye process, decreases in a very abrupt way from pure MB to 0.9 mole fraction (Table 6) which shows the strong effect of the alcohol on the MB dipolar structure, for increasing HEXol concentration first the relaxation time is almost constant and then it increases in an almost linear fashion (for $\mathrm{x}_{\mathrm{MB}}<0.5$ ), thus for HEXol rich regions an unlike dipolar interaction among both molecules may be inferred in a parallel arrangement as $\mathrm{g}>1$ shows. We may consider also the possible development of unlike hydrogen bonding among both molecules, through the carbonyl oxygen of $\mathrm{MB}$ and the hydrogen of the hydroxyl group in the alcohol, although this heteroassociation may be developed in the mixtures its population should be very low as the low relaxation times of the first Debye process show (which point to a dipolar arrangement). The behaviour of the second Debye process assigned to HEXol features shows a parallel behaviour to that of the first one, the intensity decreases upon $\mathrm{MB}$ addition in a very remarkable way, thus the hydrogen bonded chain-like network formed by alcohol molecules is clearly disrupted by the presence of $\mathrm{MB}$ molecules, the relaxation time also moves to low frequencies thus pointing to a decrease in the size of the aggregates and to an increasing population of monomers in the mixtures. Hence, the structure of these mixtures is dominated by dipolar interactions among both molecules in a parallel arrangement, although not so effective as in pure alcohol, with a weakening of hydrogen bonding among alcohol molecules and with a very low population of unlike hydrogen bonding among both molecules. The mixing static dielectric constant, Fig. 8, shows a S-shaped behaviour, being negative for MB rich mixtures and positive for alcohol rich ones, the parallel arrangement of dipoles in HEXol dominated regions produces a larger static dielectric constant and thus a positive mixing property, whereas for MB rich mixtures the parallel arrangement among unlike molecules is not so effective, g values are close to 1 , and thus the mixing property is slightly negative.

\section{MB + hexanoic acid mixtures at 298. $15 \mathrm{~K}$}

Results for this system are reported in Figs. 15-16 and in Table 7. The behaviour of pure linear carboxylic acids such as HEXac have been studied previously in the literature [31-33], the formation of dimmers through hydrogen bonding among acid molecules for the longer members of the series is well known. This association gives rise to a large correlation among dipoles because the dimmers produce a very efficient anti-parallel arrangement of dipoles and thus they are almost apolar structures which will justify the very low values of the static dielectric constant for this pure fluid, Table 7 . Thus, liquid HEXac in the pure state may be considered as a mixture of polar monomers $\left(\mu_{298.15 k}=1.92 \mathrm{D}\right.$, Table 2$)$ dispersed in a dominated medium composed by apolar dimmers; anyhow, the very low values of the static dielectric constant for HEXac points to a very low population of monomers in the fluid state, we have also been able of fitting the dielectric spectra of pure HEXac with a single Debye term which show the low effect of monomers 


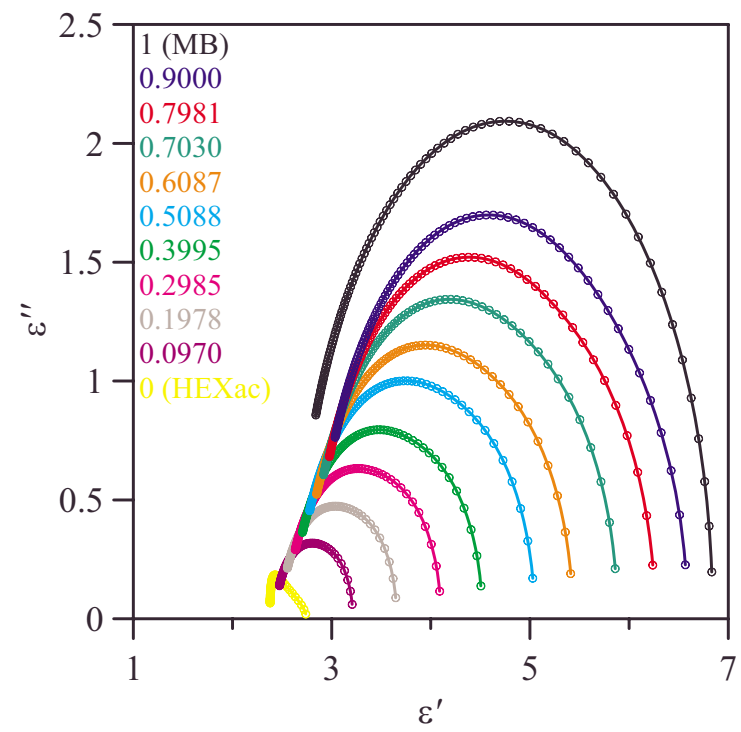

\section{Figure 15}

Cole - Cole plots of the complex dielectric function for $x M B+(I-x)$ HEXac binary mixtures for different MB mole fractions, $x$, at $298.15 \mathrm{~K}$. Real, $\varepsilon^{\prime}$, and imaginary, $\varepsilon^{\prime \prime}$, parts. Open circles, experimental points; solid lines, Debye relaxation processes (2 Debye processes for mixtures, I Debye process for pure compounds) with parameters from Table 7.
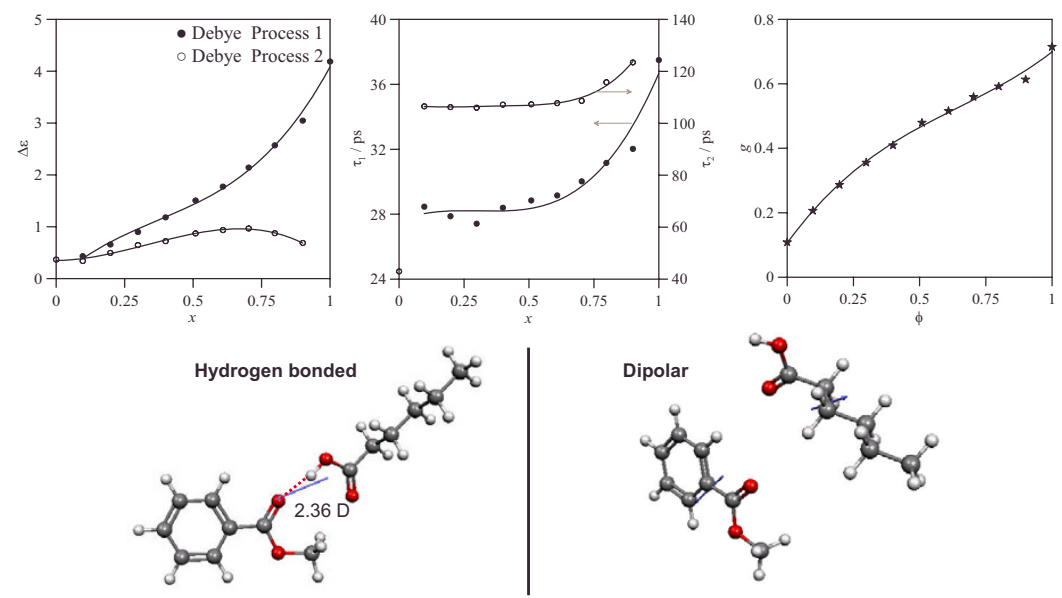

\section{Figure 16}

Dielectric strength, $\Delta \varepsilon$, and relaxation time, $\tau$, obtained from the fit of experimental dielectric spectra according to two Debye processes and Kirkwood correlation factor, g, for $x$ MB + (I - x) HEXac binary mixtures at 298.15 K. Continuous lines are plotted to show trends. Parameters from Table 7. Density data required for $g$ calculation obtained from Ref. [6]. $\varphi=\mathrm{MB}$ volume fraction. Molecules reported show the gas phase calculated structures of $\mathrm{HEXac} / \mathrm{MB}$ pairs (B3LYP/6-3।I++g** theoretical level) through hydrogen bonding and dipolar interaction. Atom color code as in Fig. 3. Blue arrows, calculated molecular dipole moments. The dipole moment reported in the hydrogen bonding panel is the theoretical value for the dimmer. 
Table 7: Parameterization of experimental dielectric spectra according to two Debye processes for mixtures and I Debye process for pure fluids for $x \mathrm{MB}+(\mathrm{I}-\mathrm{x}) \mathrm{HEXac}$ in the $200 \mathrm{MHz}-20 \mathrm{GHz}$ frequency range at 298. I5 K. Limiting highfrequency permittivity, $\varepsilon_{\infty}$, static dielectric constant, $\varepsilon_{\mathbf{s}}$, Dielectric strength, $\Delta \varepsilon$, and relaxation time, $\tau_{D}$

\begin{tabular}{ccccccc}
\hline$x$ & $\varepsilon_{\infty}$ & $\Delta \varepsilon_{1}$ & $\Delta \varepsilon_{2}$ & $\tau_{D 1} / \mathrm{ps}$ & $\tau_{\mathrm{D} 2} / \mathrm{ps}$ & $\chi^{2}$ \\
\hline 1 & 2.66 & 4.18 & - & 37.49 & - & 0.015 \\
0.9000 & 2.85 & 3.05 & 0.69 & 32.02 & 123.42 & 0.014 \\
0.7981 & 2.81 & 2.57 & 0.88 & 31.16 & 115.82 & 0.014 \\
0.7030 & 2.77 & 2.14 & 0.97 & 30.02 & 108.63 & 0.014 \\
0.6087 & 2.72 & 1.78 & 0.94 & 29.15 & 107.75 & 0.013 \\
0.5088 & 2.66 & 1.51 & 0.87 & 28.85 & 107.31 & 0.013 \\
0.3995 & 2.61 & 1.18 & 0.72 & 28.40 & 107.18 & 0.012 \\
0.2985 & 2.56 & 0.90 & 0.65 & 27.42 & 106.00 & 0.013 \\
0.1978 & 2.50 & 0.66 & 0.50 & 27.87 & 106.23 & 0.010 \\
0.0970 & 2.44 & 0.43 & 0.34 & 28.46 & 106.53 & 0.011 \\
0 & 2.38 & - & 0.37 & - & 42.98 & 0.010 \\
\hline
\end{tabular}

on the whole dielectric fluid behaviour. The Kirkwood correlation factor for pure HEXac is remarkably low, Fig. 16, which shows the high degree of association among these molecules and discards the existence of noticeable populations of non-associated polar monomers. The $g$ factor in the mixture decreases from the highest value corresponding to pure MB upon HEXac addition, thus the anti-parallel dipolar arrangement in pure $\mathrm{MB}$ is reinforced with the acid addition, Fig. 16. Hence, although the dipolar interaction among like MB molecules is weakened upon mixing, a new dipolar interaction among unlike molecules appears, in which the anti-parallel dipolar disposition is even more remarkable, compared with that in pure $\mathrm{MB}$, thus an important unlike dipolar interaction should be present in the mixture, Fig. 16. We may think even in the formation of unlike pairs through hydrogen bonding among both molecules, Fig. 16; this situation gives rise to a pair with a remarkable dipolar moment and thus it should be a factor tending to increase the mixture dipole moment and thus the $g$ factor, and also the static dielectric constant, as this decreases remarkably upon mixing we should conclude that if these hydrogen bonded pairs are present in the mixture, their population should be very small compared with the prevailing dipolar anti-parallel pairs of like and unlike nature.

The first Debye process in the mixture assigned to $\mathrm{MB}$ features decreases its amplitude upon HEXac addition first in a steep way and then in a smooth linear fashion, the relaxation time also moves to lower times but for $\mathrm{xMB}<0.5$ it remains almost constant. Thus MB molecules in dipolar unlike associates relax at lower times which points to a weaker interaction among unlike that like molecules as we may expect. The second Debye process show a complex behaviour, its relaxation time shows a trend almost parallel to that of the first process although at larger times whereas its amplitude is almost constant in the studied range. Hence, a remarkable population of HEXac dimmers should be present in the mixtures even for relatively high MB concentrations, MB molecules interact with acid ones through dipolar interaction but HEXac also tends to keep its like hydrogen bonding structure. Finally, the mixing static dielectric constant, Fig. 8, is slightly positive in the whole composition range, although dipolar heteroasociates are also formed in an anti-parallel arrangement, this is not so effective as in pure acid dimmers for which an almost cancellation of dipoles is obtained ( $\mathrm{g}=0.11$ for pure HEXac, Table 7 ), thus a slight excess of 
dipoles is obtained upon mixing which will justify the positive small mixing property. Anyhow, the small values of the mixing static dielectric constant points to a very efficient interaction among ester and acid molecules.

\section{MB + diisopropyl ether mixtures at 298.15 K}

The dielectric spectra obtained for this binary system is reported in Fig. 17, the results reported show that as ISOP mole fraction increases the maxima of the relaxation peaks shift toward lower times and for high ISOP concentrations the peaks are not fully included in our frequency range, thus the high frequency permittivities reported in Table 8 obtained from the fits to Debye-type functions should be considered with caution. ISOP is a molecule with an almost globular shape and a high degree of symmetry, Fig. 18, it has a low dipole moment (1.33 D, Table 2) placed in the alkoxy oxygen which is almost in the center of the molecule, this fact hinders the interactions among neighbour molecules as the Kirkwood factor slightly greater than 1 shows, Fig. 18. The population of monomers in pure ISOP should be remarkable as the low relaxation time shows; anyhow, a certain dipolar weak interaction among ISOP molecules is developed in the pure state, these should be in a parallel arrangement $(g>1)$ and thus a slight reinforcement of the dipolar moment may be expected which would justify the value of the static dielectric constant, Table 8. The low value of ISOP density $\left(0.71807 \mathrm{~g} \mathrm{~cm}^{-3}\right.$ at $298.15 \mathrm{~K}$, Table 2) [6] shows an ineffective packing of ISOP molecules in which free volume should be remarkable [6]; hence, upon MB addition, ester molecules fit in the free spaces of ISOP dominated fluid, as the remarkably nega-

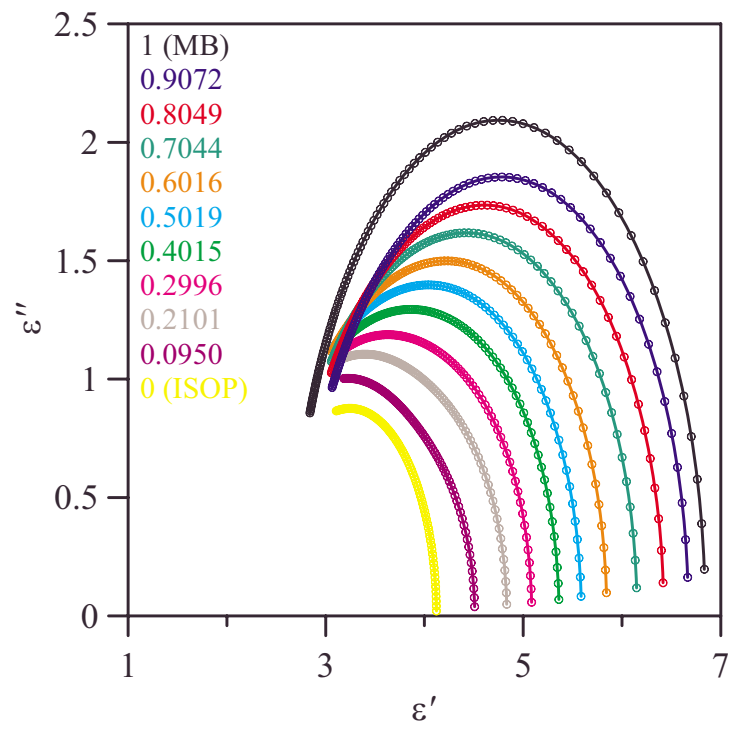

\section{Figure 17}

Cole - Cole plots of the complex dielectric function for $x \mathrm{MB}+(\mathrm{I}-\mathrm{x})$ ISOP binary mixtures for different MB mole fractions, $x$, at $298.15 \mathrm{~K}$. Real, $\varepsilon^{\prime}$, and imaginary, $\varepsilon^{\prime \prime}$, parts. Open circles, experimental points; solid lines, Debye relaxation processes (2 Debye processes for mixtures, I Debye process for pure compounds) with parameters from Table 8. 
Table 8: Parameterization of experimental dielectric spectra according to two Debye processes for mixtures and I Debye process for pure fluids for $x \mathrm{MB}+(I-x)$ ISOP in the $200 \mathrm{MHz}-20 \mathrm{GHz}$ frequency range at 298. $15 \mathrm{~K}$. Limiting highfrequency permittivity, $\varepsilon_{\infty}$, static dielectric constant, $\varepsilon_{\mathbf{s}}$, Dielectric strength, $\Delta \varepsilon$, and relaxation time, $\tau_{\mathrm{D}}$

\begin{tabular}{ccccccc}
\hline$x$ & $\varepsilon_{\infty}$ & $\Delta \varepsilon_{1}$ & $\Delta \varepsilon_{2}$ & $\tau_{\mathrm{DI}} / \mathrm{ps}$ & $\tau_{\mathrm{D} 2} / \mathrm{ps}$ & $\chi^{2}$ \\
\hline 1 & 2.66 & 4.18 & - & 37.49 & - & 0.015 \\
0.9072 & 2.75 & 3.23 & 0.69 & 37.14 & 14.12 & 0.016 \\
0.8049 & 2.68 & 2.63 & 1.12 & 36.05 & 14.74 & 0.016 \\
0.7044 & 2.60 & 2.21 & 1.34 & 34.35 & 13.72 & 0.014 \\
0.6016 & 2.50 & 2.03 & 1.35 & 30.87 & 11.43 & 0.014 \\
0.5019 & 2.42 & 1.77 & 1.43 & 29.00 & 10.39 & 0.013 \\
0.4015 & 2.38 & 1.47 & 1.52 & 27.82 & 9.31 & 0.014 \\
0.2996 & 2.30 & 1.17 & 1.62 & 27.51 & 8.44 & 0.012 \\
0.2101 & 2.22 & 0.89 & 1.72 & 29.00 & 7.63 & 0.011 \\
0.0950 & 2.19 & 0.58 & 1.73 & 31.43 & 7.24 & 0.010 \\
0 & 2.37 & - & 1.79 & - & 9.41 & 0.011 \\
\hline
\end{tabular}

tive excess molar volume shows [6]. A certain dipolar unlike interaction seems to be developed although it should be weak because the shapes of both molecules hinder it. Anyhow, the $g$ values close to unity, greater than one for ISOP dominated regions or lower than 1 for MB dominated ones, discards the existence of strong dipolar correlations and probably the mixture structure is dominated by steric effects.

The first Debye process originated from MB features decreases its amplitude upon ISOP addition with its relaxation time decreasing also but going trough a minimum at very high ISOP concentrations, the second process shows a very similar trend. Thus, upon ISOP addition to pure MB the dipolar structure of like $\mathrm{MB}$ associates is disrupted by the ether molecules, the development of unlike associates is not very remarkable and if they are present they do in a parallel arrange-
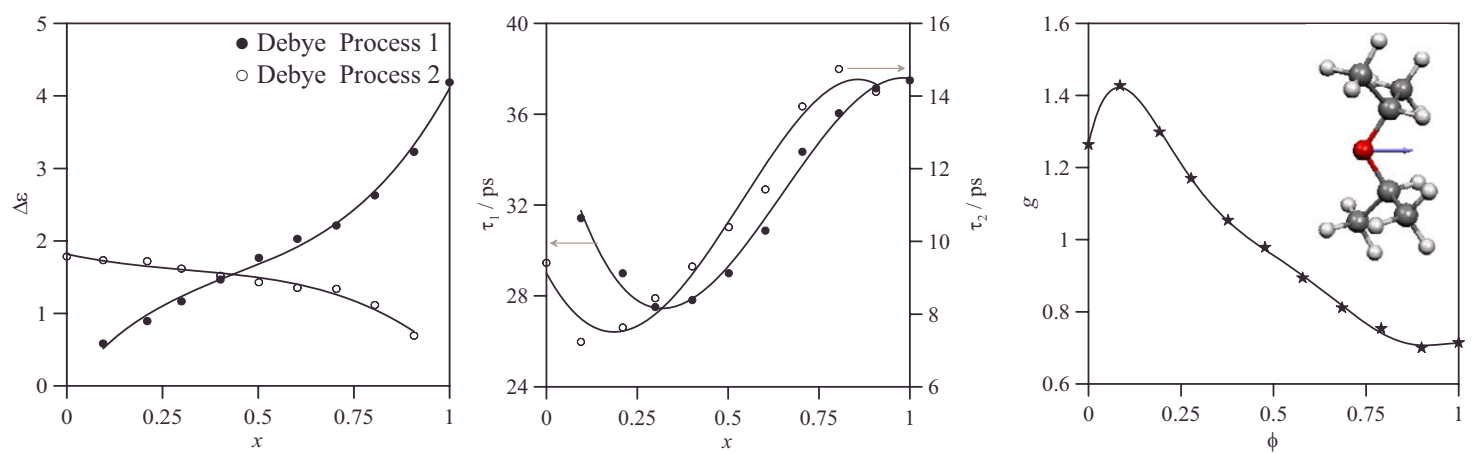

Figure 18

Dielectric strength, $\Delta \varepsilon$, and relaxation time, $\tau$, obtained from the fit of experimental dielectric spectra according to two Debye processes and Kirkwood correlation factor, g, for $x$ MB + (I - x) ISOP binary mixtures at $298.15 \mathrm{~K}$. Continuous lines are plotted to show trends. Parameters from Table 8. Density data required for $g$ calculation obtained from Ref. [6]. $\varphi=$ MB volume fraction. Molecule inside $g$ factor panel show the gas phase calculated structures of ISOP (B3LYP/6-3II++g** theoretical level). Atom color code as in Fig. 3. Blue arrows, calculated molecular dipole moment $(\mu=1.33 \mathrm{D})$. 
ment, thus the effective dipole moment in the mixture should increase giving rise to a slightly positive static dielectric constant, Fig. 8. As the ISOP mole fraction increases, ester molecules are diluted in ISOP dominated liquid free spaces in which the development of unlike dipolar interactions seems to be not very efficient because of the distance among both dipoles. The relaxation time of the second process rising from ISOP features moves to slightly larger times which points to a slightly increasing population of unlike associates although in any case it should be low.

\section{Conclusion}

The structure of pure aromatic esters and of $\mathrm{MB}$ containing binary mixtures has been studied by microwave dielectric spectroscopy. The relaxational behaviour of the studied systems has been fitted properly according to Debye-type processes which parameters have been analyzed according to molecular level features. Pure esters show a remarkable dipolar ordering in an anti-parallel way which decreases with increasing chain length or temperature. The structure of mixed solvents is strongly dependent on the co-solvent properties but we may conclude that it is of dipolar nature in all the cases. Anti - parallel dominating dipolar interactions are inferred for most of the systems, only for HEXol systems a parallel arrangement is preferred. Upon mixing, the dipolar structure of $\mathrm{MB}$ is weakened in most of the cases and only with molecules such as HEXol or HEXac a remarkable unlike dipolar interaction is inferred. Anyhow, very subtle changes happens upon mixing, specially for apolar co-solvents such as HEX or cHEX, only for alcohols or carboxylic acids more remarkable effects are present although for these systems unlike hydrogen bonding should be discarded.

\section{Appendix}

\section{Abbreviations and symbols}

BB butylbenzoate

cHEX cyclohexane

clHEX 1-chlorohexane

DRS Dielectric relaxation spectroscopy

$M$ molar mass

EB ethylbenzoate

$g$ Kirkwood correlation factor

HEX n-hexane

HEXac hexanoic acid 
HEXol 1-hexanol

ISOP diisopropyl ether

MB methylbenzoate

PA phenylacetate

PB propylbenzoate

$x$ mole fraction

\section{Greek symbols}

$\Delta \varepsilon_{i}$ relaxation amplitude

$\varepsilon^{\prime}$ real part of the complex dielectric function

$\varepsilon^{\prime \prime}$ imaginary part of the complex dielectric function

$\varepsilon^{*}$ complex dielectric function

$\varepsilon_{\infty}$ dielectric constant in the high frequency limit

$\varepsilon_{s, \text { mix }}$ mixing static dielectric constant

$\varepsilon_{0}$ dielectric constant in vacuum

$\varphi$ volume fraction

$\mu$ gas phase dipole moment

$v$ frequency

$\rho$ density

$\tau_{i}$ relaxation time

\section{Acknowledgements}

The financial support by Junta de Castilla y León, Project BU020A07, and Ministerio de Educación y Ciencia, Project CTQ2005-066I I/PPQ, Spain, is gratefully acknowledged. 


\section{References}

I. Kremer F, Schönhals A: Broadband Dielectric Spectroscopy. Springer 2002.

2. Sato T, Buchner R: J Mol Liq 2005, I I 7:23-3I.

3. Grochulski T, Kocot A: Mol Phys 1994, 81:705-716.

4. Aparicio S, Alcalde R, Dávila MJ, García B, Leal JM: J Phys Chem B 2007, I I I:44 I 7-443 I.

5. García B, Alcalde R, Aparicio S, Leal JM: Ind Eng Chem Res 2002, 4 I:4399-4408.

6. García B, Alcalde R, Aparicio S, Leal JM: Phys Chem Chem Phys 2002, 4:5833-5840.

7. García B, Aparicio S, Alcalde R, Leal JM: J Phys Chem B 2003, 107:13478-I 3486.

8. García B, Aparicio S, Navarro AM, Alcalde R, Leal JM: J Phys Chem B 2004, I 08: I 584I-I 5850.

9. Aparicio S, Alcalde R, Leal JM, García B: J Phys Chem B 2005, 1 09:6375-6385.

10. Aparicio S, Alcalde R, García B, Leal JM: Ind Eng Chem Res 2005, 44:7575-7583.

II. Alcalde R, Aparicio S, García B, Leal JM: J Phys Chem B 2005, 109:19908-199|4.

12. Fröhlich H: Theory of Dielectrics Oxford University Press; 1949.

13. Franck EU, Deul R: Faraday Discuss Chem Soc 1978, 66:191-198.

14. Ghanadzadeh A, Mamaghani M, Anbir L: J Sol Chem 2003, 32:625-636.

15. Vogel H: Phys Z 192I, 22:645-646.

16. Tamman D, Hesse Z: Z Anorg Allg Chem 1926, I 56:245-247.

17. Fulcher GS: J Am Ceram Soc 1925, 8:339-355.

18. Barthel J, Buchner R, Wurn B: J Mol Liq 2002, 98-99:5 I-69.

19. Angell CA, Bressel RD: J Phys Chem 1978, 82:2622-1629.

20. Balamurugan D, Kumar S, Krishnan S: J Mol Liq 2005, I 22: I I-I4.

21. Chaudhari A, Chaudhari HC, Mehrota SC: Bull Korean Chem Soc 2004, 25: I 403-I 407.

22. Reid C, Prausnitz JM, Poling BE: The Properties of Gases and Liquids McGraw - Hill; 1987.

23. Rowlinson JS, Swinton FL: Liquids and Liquid Mixtures. Butterworths 1982.

24. Bolotnikov MF, Neruchev YA: J Chem Eng Data 2004, 49:895-898.

25. Garg SK, Smyth CP: J Phys Chem 1965, 69:1294-I30I.

26. Feldman YD, Levin VV: Chem Phys Lett 1982, 85:528-530.

27. Feldman Y, Andrianov A, Polygalov E, Ermolina I, Romanychev G, Zuev Y, Milgotin B: Rev Sci Instrum 1996, 67:3208-32I6.

28. Petong P, Pottel R, Kaatze U: J Phys Chem A 1999, I 03:6 I 14-6121.

29. Garg KS, Smyth CP: J Phys Chem 1965, 69:1294-I30I.

30. Barthel J, Bachhuber K, Buchner R, Hetzenauer H: Chem Phys Lett 1990, 165:369-373.

31. Mognaschi ER, Chierico A: Z Naturf A 1986, 41:49I.

32. Mognaschi ER, Zullino L, Chierico A: J Phys D Appl Phys 1984, 17:1007-1012.

33. Mognaschi ER, Laboranti LM, Chierico A: J Phys II I993, 3:127I-I 277. 